\title{
Fördert eine videobasierte Intervention im Praktikum die professionelle Wahrnehmung von Klassenführung? - Eine quantitativ-inhaltsanalytische Messung von Subprozessen professioneller Wahrnehmung
}

\author{
Kira Elena Weber • Christopher Neil Prilop • Stephan Viehoff • \\ Bernadette Gold • Marc Kleinknecht
}

Online publiziert: 1. April 2020

(C) Der/die Autor(en) 2020

Zusammenfassung In der Lehrerinnen- und Lehrerbildung werden zunehmend videogestützte Lerngelegenheiten eingesetzt, um die professionelle Wahrnehmung von Unterricht zu fördern. In der vorliegenden Studie wurde ein quantitativ-inhaltsanalytisches Verfahren gewählt, um zu überprüfen, ob sich die professionelle Wahrnehmung von Klassenführung durch eine videobasierte Intervention im Praktikum fördern lässt. Die Interventionsgruppe (IG, $n=26)$ analysierte während eines vierwöchigen Unterrichtspraktikums zwei eigene Videosequenzen und erhielt videobasiertes Peer- und Expertenfeedback, während die Kontrollgruppe (KG, $n=26$ ) eine konventionelle Praktikumsbegleitung mit face-to-face Feedback durchlief. Beide Gruppen absolvierten einen Prä-Post-Test und beantworteten offene Fragen zu einer Videosequenz. Die Antworten wurden inhaltsanalytisch ausgewertet und Unterschiede zwischen den Gruppen mit Hilfe non parametrischer Verfahren überprüft. Die Ergebnisse zeigen, dass sich die IG in fast allen Bereichen der wissensgesteuerten Verarbeitung von Unterricht verbessert sowie in einem Bereich der selektiven

Dr. K. E. Weber $(\bowtie) \cdot$ C. N. Prilop · S. Viehoff · Prof. Dr. M. Kleinknecht

Institut für Bildungswissenschaften, Leuphana Universität Lüneburg,

Universitätsallee 1, 21335 Lüneburg, Deutschland

E-Mail: kweber@leuphana.de

C. N. Prilop

E-Mail: prilop@leuphana.de

S. Viehoff

E-Mail: stephan-viehoff@web.de

Prof. Dr. M. Kleinknecht

E-Mail: marc.kleinknecht@leuphana.de

Jun.-Prof. Dr. B. Gold

Professionsentwicklung und Didaktik der Lehrerbildung, Universität Erfurt, Nordhäuser Straße 63, 99089 Erfurt, Deutschland

E-Mail: bernadette.gold@uni-erfurt.de 
Wahrnehmung, während sich die KG lediglich im Bereich der Klassifikation von Wissen verbessert.

Schlüsselwörter Intervention · Lehreramtsstudierende · Praktikum · Professionelle Wahrnehmung $\cdot$ quantitativ-inhaltsanalytisch

\title{
Does a video-based practicum intervention provide a realistic picture of classroom management? A quantitative content analysis of the subprocesses of professional awareness
}

\begin{abstract}
Video-based learning opportunities are increasingly being used to promote professional awareness in teacher education. In the current study, a quantitative content analysis was conducted to ascertain whether professional awareness of classroom management can be promoted through a video-based intervention in a teaching practicum. During a four-week teaching practicum, the intervention group (IG, $n=26$ ) analyzed two separate video sequences and received video-based peer and expert feedback, while the control group ( $\mathrm{CG}, n=26)$ participated in a conventional practicum setting with face-to-face feedback. Both groups completed a prepost-test and answered open-ended questions about a video sequence. The answers were analyzed using content analysis and differences between the groups were examined with non-parametric methods. The results show that the IG improved in almost all areas of knowledge-based reasoning and in one area of noticing, whereas the $\mathrm{CG}$ improved only in the area of knowledge classification.
\end{abstract}

Keywords Intervention - Knowledge-based reasoning $\cdot$ Noticing · Practicum · Preservice teachers · Professional vision - Quantitative content analysis

\section{Einleitung}

Im Rahmen der Lehrerinnen- und Lehrerbildung stellt der Erwerb professioneller Unterrichtswahrnehmung die Grundlage wissens- und evidenzbasierter Reflexion eigener Praxis dar und kann als Vorstufe professioneller Handlungskompetenz angesehen werden (vgl. Stürmer 2011; Blömeke et al. 2014; Jahn et al. 2014). Professionelle Wahrnehmung wird als wissensbasierter Prozess verstanden, der die Komponenten selektive Wahrnehmung/noticing (wissensbasierte Prozesse der Aufmerksamkeitssteuerung) und knowledge-based reasoning (wissensgesteuerte Verarbeitung von Unterricht) umfasst (van Es und Sherin 2008). In verschiedenen Studien wird knowledge-based reasoning auch als Interpretieren relevanter Unterrichtssituationen beschrieben, wobei unterschiedliche Aspekte des Interpretierens (z. B. Beschreiben, Erklären, Vorhersagen, Handlungsalternativen; Santagata und Guarino 2011; Seidel und Stürmer 2014; Beschreiben, Erklären, Bewerten; Seidel und Prenzel 2007; Schwindt 2008) unterschieden werden. Barth (2017) unterscheidet in ihrem Kompetenzmodell zum professionellen Wahrnehmen und Handeln im Unterricht die Kompetenzfacetten Wissen, Erkennen, Beurteilen, Generieren, Entscheiden und Implementieren, während beispielsweise Janik et al. (2013) folgende Subprozesse der 
professionellen Wahrnehmung operationalisieren: Wahrnehmen, Beschreiben, Interpretieren, Erklären, Vorhersagen, Bewerten und Handlungsalternativen vorlegen. Hier zeigt sich, dass es bislang keine einheitliche Definition der Subprozesse der professionellen Wahrnehmung von Unterricht gibt. Allen Ansätzen liegt jedoch die Annahme zugrunde, dass Lehrkräfte zunächst lernprozessrelevante Situationen im Unterrichtsgeschehen wahrnehmen müssen (selektive Wahrnehmung), um diese dann unter Einbezug theoretischer Wissensbestände (Jahn et al. 2014) zu beschreiben, zu interpretieren und Handlungsalternativen zu generieren.

Der systematische Aufbau professioneller Wahrnehmungs- und Handlungskompetenz soll dabei über eine möglichst direkte Theorie-Praxis-Verknüpfung erfolgen (Stürmer 2011). Für Studierende bietet sich als Gegenstand der Theorie-Praxis-Verbindung die Klassenführung an, da es Novizinnen und Novizen häufig schwer fällt eine effektive Klassenführung während ihrer ersten Unterrichtsversuche zu realisieren (Seidel und Stürmer 2014; Wolff et al. 2017). Eine Förderung der professionellen Wahrnehmung von Klassenführung (PWKF) sollte daher zu einem frühen Zeitpunkt des Studiums erfolgen (Hellermann et al. 2015).

Gefördert werden kann ein anwendungsorientierter Wissenserwerb durch situiertes Lernen (Blömeke 2002). Dabei wird zunehmend auf videogestützte Lerngelegenheiten zurückgegriffen (Kleinknecht und Gröschner 2016), da sie die Möglichkeit des wiederholten Betrachtens bieten und eine Distanz zum Geschehen schaffen, die eine multiperspektivische Betrachtung ohne Handlungsdruck ermöglicht (Kramer et al. 2017). Unterrichtsvideos zeichnen sich zudem durch einen hohen Anteil an Authentizität und Praxisnähe aus und lassen sich in unterschiedlicher Form (eigene vs. fremde Videos) und in verschiedenen Lernumgebungen einsetzen. Unterrichtsvideos bieten jedoch nicht nur die Gelegenheit, die professionelle Wahrnehmung angehender Lehrkräfte zu fördern; sie stellen auch ein wertvolles Instrument der Kompetenzmessung dar (Seidel und Prenzel 2007). Videos dienen als Stimulus um professionelles Wissen in authentischen Unterrichtssituationen sicht- und messbar machen (Seidel und Thiel 2017). Videobasierte Testmethoden ermöglichen somit handlungsorientierte Wissensbestände zu berücksichtigen und sie valide, reliabel und objektiv zu erfassen (Lindmeier 2013). Die vorliegenden videobasierten Verfahren zur Messung der professionellen Wahrnehmung unterscheiden sich dabei in ihrem Grad der Standardisierung. In hoch standardisierten Verfahren werden z. B. Ratingskalen verwendet, die mit einem Masterrating abgeglichen werden (u. a. Seidel et al. 2010; Gold und Holodynski 2017), während weniger standardisierte Verfahren offene Aufgabenformate oder Fragen verwenden, die anschließend kodiert werden (u.a. Sherin und van Es 2009; Barth 2017). Je nach Zielsetzung kann es dabei notwendig sein, weniger standardisierte Verfahren $\mathrm{zu}$ wählen und dadurch tiefergehende Erkenntnisse zu gewinnen als dies durch hoch standardisierte Verfahren möglich ist (Lindmeier 2013). In vorliegenden standardisierten Instrumenten zur Erfassung der professionellen Unterrichtswahrnehmung werden bislang die Subprozesse Beschreiben, Erklären und Vorhersagen (Seidel et al. 2010) oder Beschreiben und Interpretieren (Gold und Holodynski 2017) gemessen. Die Prozesse Wahrnehmen und Handlungsalternativen vorlegen werden bislang durch standardisierte Verfahren zur Erfassung der professionellen Unterrichtswahrnehmung nicht abgedeckt. Des Weiteren legen standardisierte Messinstrumente im Vorhinein fest, 
was genau beobachtet werden soll (Lamnek 1995), während offene Messinstrumente den „Wahrnehmungstrichter“ offen lassen und mehr Raum für eigene Deutungen schaffen (Lamnek 1995, S. 22). Barth (2017) betont zudem, dass durch geschlossene Antwortformate nur spezifisches Vorwissen aktiviert wird und geschlossene Items stärker die Möglichkeit des Ratens zulassen.

In einer aktuellen Studie von Weber et al. (2018) konnte gezeigt werden, dass sich die PWKF bei Lehramtsstudierenden durch eine online- und videobasierte Feedbackintervention im Praktikum fördern lässt. Studierende, die videobasiertes Peer- und Expertenfeedback während des Praktikums erhielten, verbesserten ihre professionelle Wahrnehmung in den Bereichen Allgegenwärtigkeit und Strukturierung signifikant mehr als Studierende die ausschließlich face-to-face Peer- und Expertenfeedback erhielten. In der Studie wurde ein hoch standardisiertes Verfahren zur Messung der PWKF (Gold und Holodynski 2017) genutzt. Die standardisierten Items beziehen sich auf die Beschreibung und Interpretation von Unterrichtssituationen. Darüber hinaus umfasst der videobasierte Test auch offene Fragen, da Gold und Holodynski (2017)argumentieren, dass einige Subprozesse der PWKF nicht durch geschlossene Items erfasst werden können. Insbesondere der Subprozess Handlungsalternativen vorlegen kann über standardisierte Items nicht erfasst werden, da hierbei konkrete Handlungsalternativen vorgeschlagen und von den Probanden bewertet werden müssten. Dasselbe gilt für die Bewertung, da die geschlossenen Items bereits vorgeben was und wie bewertet werden soll (Barth 2017). Auch die selektive Wahrnehmung kann durch standardisierte Items nur unzureichend gemessen werden, da der Itemtext bereits den Fokus der Wahrnehmung vorgibt. Dasselbe gilt für die Klassifikation der deklarativen Wissensbestände, als Kriterium einer kompetenten Unterrichtswahrnehmung (Schwindt 2008; Blomberg et al. 2011), da in geschlossenen Items Einzelereignisse bereits in übergeordnete Konzepte eingeordnet sind. Durch eine Auswertung der Antworten auf die offenen Fragen sollen in der vorliegenden Studie die Ergebnisse der standardisierten Items entsprechend um die Dimension der selektiven Wahrnehmung und um die Subprozesse Bewerten und Handlungsalternativen vorlegen erweitert werden. Zudem soll überprüft werden, ob sich die Klassifikation der deklarativen Wissensbestände zum Thema Klassenführung durch die videobasierte Intervention verändern.

\subsection{Klassenführung}

Die Bedeutung effektiver Klassenführung als ein zentraler Bedingungsfaktor gelingenden Unterrichts wurde vielfach bestätigt (Kramer et al. 2017). Klassenführung lässt sich definieren als die vielfältigen Aktionen einer Lehrperson, die darauf ausgerichtet sind, Ziele des Unterrichtens und Lernens zu etablieren, zu unterstützen und zu erleichtern, damit Lehr- und Lernprozesse in der Schule möglichst reibungslos ablaufen (Helmke und Helmke 2014). In Anlehnung an Kounin (1976) leiten Hellermann et al. (2015) drei zentrale, sich ergänzende Aspekte einer präventiven und proaktiven Steuerung von Unterrichtsaktivitäten ab: Monitoring (Allgegenwärtigkeit), prozessuale Strukturierung und die Etablierung von Regeln und Routinen. Monitoring beschreibt die Fähigkeit der Lehrperson, alle relevanten Vorgänge des Unterrichts wahrzunehmen, auch wenn sie gerade nur mit einem Teil der Klas- 
se direkt interagiert (Schönbächler 2008) und ihre Aufmerksamkeit simultan auf mehrere Ereignisse des Unterrichtsgeschehens zu richten. Studien zum Monitoring verdeutlichen die unterschiedlichen Wahrnehmungs- und Handlungsmuster von Expertinnen und Experten gegenüber Novizinnen und Novizen. Während Expertinnen und Experten ihre Aufmerksamkeit vornehmlich auf die typischen Elemente einer Situation lenken, fokussieren Novizinnen und Novizen besonders auffällige Merkmale (Thiel et al. 2012). Die prozessuale Strukturierung spielt eine zentrale Rolle bei der Steuerung von Unterricht (Hellermann et al. 2015). Nach Hellermann et al. (2015) verläuft strukturierter Unterricht schwungvoll, motiviert die Klasse zum Lernen und zeichnet sich durch reibungslose Übergänge zwischen zwei Unterrichtsaktivitäten aus. Diese Definition beschreibt die Ablaufsteuerung der Lektionen und die Gestaltung der Phasenübergänge (Schönbächler 2008) und greift die Aspekte Reibungslosigkeit und Schwung, Gruppenmobilisierung sowie das Übergangsmanagement aus Kounins (1976) Prinzipien effizienter Klassenführung auf. Die Aspekte Monitoring und prozessuale Strukturierung verdeutlichen, dass effektive Klassenführung darauf ausgerichtet ist, präventive Ordnungsstrukturen zu initiieren, damit Unterricht möglichst störungsarm abläuft. Der Einführung von Regeln und Routinen fällt in der Vermeidung von Störungen eine besondere Rolle zu (Seidel 2009). Ziel der Vereinbarung von Regeln und Routinen ist es, dem Unterrichtsgeschehen ,einen klaren Handlungsrahmen zu geben, der das Zusammenleben und Lernen organisiert“" (Hellermann et al. 2015, S. 98).

Alle Aktivitäten der Klassenführung haben die Funktion, die unterschiedlichen Handlungsimpulse der Schülerinnen und Schüler in den konkreten Unterrichtssituationen auf ein bestimmtes Handlungsprogramm (Unterrichtsplanung; Barth 2017) hin auszurichten, das die Agenda für den Verlauf des Unterrichts darstellt (Thiel et al. 2012). Um diese aufrechtzuerhalten ,muss die Lehrkraft einen (primären) Handlungsvektor etablieren“ (Barth 2017, S. 2). Besonders in Phasenübergängen stellt dies nach Thiel et al. (2012) eine besondere Schwierigkeit dar, da hier der Übergang organisiert und die Anschlussfähigkeit sichergestellt werden muss. Phasenübergänge im Unterricht bergen oft das Risiko unvorhergesehener Unterrichtsstörungen und erfordern in einem hohen Maß mehrere Ereignisse simultan und überlappend zu organisieren. Um das Zusammenspiel einzelner Maßnahmen und Interventionen in Handlungsübergängen zu untersuchen, haben Thiel et al. (2012) mittels einer Experten-Novizen-Studie eine sequenzanalytische Rekonstruktion von Strategien und Techniken des Klassenmanagements in Übergangssituationen vorgenommen. Dabei haben sie den Fokus bewusst auf Übergänge zwischen Aktivitätsstrukturen gelegt, da dort grundlegende Anforderungen des Klassenmanagements in kondensierter Form sichtbar werden und sich die deklarative Wissensbasis von Novizen und Experten besonders stark unterscheidet. Drei wesentliche Anforderungsmerkmale wurden segmentiert - Beendigung des primären Handlungsvektors, Etablierung und Stabilisierung des neuen Handlungsvektors. Zusätzlich wird der Umgang mit Unterrichtsstörungen um den Umgang mit konkurrierenden Handlungsvektoren vervollständigt. Im Umgang mit solchen konkurrierenden Handlungsvektoren lassen sich große Diskrepanzen zwischen Experten- und Novizenhandeln feststellen (Thiel et al. 2012). Entsprechend sollten Studierende bereits in ihrer Ausbildung lernen, wesentliche 
Aspekte von Klassenführung selektiv wahrzunehmen und wissensgesteuert zu verarbeiten.

\subsection{Professionelle Unterrichtswahrnehmung von Klassenführung}

Lehrkräfte benötigen professionelle Kompetenzen um qualitativ hochwertigen Unterricht zu gewährleisten (Kunter et al. 2013). In dem Modell von Blömeke et al. (2015) spiegelt sich professionelle Kompetenz in Dispositionen (Wissen und affektiv-motivationale Komponenten), situationsspezifischen Fähigkeiten (Wahrnehmung, Interpretation, Entscheidung) und letztlich der unterrichtlichen Performanz wieder. Situationsspezifische Fähigkeiten wie die professionelle Wahrnehmung von Klassenführung sind somit notwendige Prozesse für professionelles Unterrichtshandeln. Hellermann et al. (2015) definieren professionelle Unterrichtswahrnehmung von Klassenführung als die Fähigkeit, „klassenführungsspezifische Unterrichtsereignisse hinsichtlich der oben genannten Facetten (1) Monitoring, (2) prozessuale Strukturierung und (3) Etablierung von Regeln und Routinen zu erkennen, deren Relevanz für den Unterrichtsprozess einzuschätzen und theoriegeleitet interpretieren zu können“ (S. 98). Die selektive Wahrnehmung bezieht sich dabei auf die Identifikation lernprozessrelevanter Interaktionen, Ereignisse und Abläufe im Unterrichtsgeschehen (Seidel et al. 2010). Da Unterrichtsgeschehen komplex und simultan abläuft (Sherin 2007), muss die Lehrkraft unwichtige Dinge ausblenden und den Fokus auf relevante Aspekte legen (van Es und Sherin 2008). Aus den wahrgenommenen Situationen werden, auf Grundlage professionellen Wissens, Schlussfolgerungen für das weitere Handeln und den weiteren Verlauf gezogen. Dabei lassen sich verschiedene Arten des Umgangs mit den wahrgenommenen Informationen unterscheiden (Beschreiben, Interpretieren, Bewerten und Handlungsalternativen vorlegen). Anhand der Ausprägung dieser Subdimensionen lassen sich Aussagen darüber treffen, inwieweit unterrichtsrelevantes Wissen bei Lehrkräften mental repräsentiert ist und in welchem Maße im Unterrichtsgeschehen auf dieses Wissen zurückgegriffen werden kann (Seidel et al. 2010; Lindmeier 2013). Novizinnen und Novizen operieren vorwiegend auf der Ebene des Beschreibens (Seidel und Prenzel 2007). Diese Beschreibungen fallen aufgrund der fehlenden theoretischen Fundierung häufig undifferenziert und übergeneralisierend aus (Stürmer et al. 2015). Personen, die auf der Ebene des Beschreibens agieren, sind seltener in der Lage, relevante Unterrichtssituationen zu identifizieren und ihre Effekte zu erklären (Seidel et al. 2010, Stürmer 2011). Zudem zeigte sich, dass Novizinnen und Novizen Ereignisse im Unterricht bereits auf Grundlage einer einfachen Beschreibung bewerten, während Expertinnen und Experten mehr Informationen bei der Bewertung mit einfließen lassen (Schwindt 2008). Ein erklärender bzw. interpretierender Umgang mit Unterrichtsbeobachtungen zeugt entsprechend bereits von einem höheren Anwendungsniveau, da theoretisches Wissens genutzt wird (Seidel et al. 2010). Van Es und Sherin (2008) messen der Interpretation zudem besonderen Wert bei, da über die wissensgestützte Interpretation wahrgenommener Unterrichtssituationen Sinnzusammenhänge konstruiert und reflektiert werden können. Hierfür benötigen Lehrkräfte bestehende Wissensstrukturen in die sie die wahrgenommenen Einzelereignisse integrieren können (Schwindt 2008). Um sicherzustellen, dass Lehrpersonen über dieselben 
Merkmale sprechen, sollten die Konzepte zudem mit zutreffenden professionstheoretischen Begriffen bezeichnet werden. Schwindt (2008) nennt diese Kompetenz Klassifikation der deklarativen Wissensbestände und beschreibt diese als weiteres Kriterium einer kompetenten Unterrichtswahrnehmung. Insbesondere im Rahmen des Praktikums und damit einhergehender Reflexions- und Feedbackgespräche erscheint die Klassifikation der deklarativen Wissensbestände daher als wichtiger Bestandteil professioneller Wahrnehmung. Nach Barth (2017) gehört Klassifikation der deklarativen Wissensbestände zur Kompetenzfacette Wissen und bildet damit die Grundlage des Kompetenzmodells zum professionellen Wahrnehmen und Handeln im Unterricht. Sowohl das Generieren von Handlungsalternativen oder das Antizipieren von Handlungsverläufen ist nach Bromme (1992) wissensabhängig. Aus der Expertiseforschung ist zudem bekannt, dass sich Experten durch einen hohen Vernetzungsgrad des Wissens mit vielfachen relationalen Verknüpfungen auszeichnen (u. a. Berliner 2001). Charakteristisch für die Kompetenz von Lehrkräften mit hoher Expertise sind vor allem eine schnell ablaufende, kategorial bewertende und trotzdem holistische Wahrnehmung von Unterrichtssituationen und eine gleichzeitig ablaufende Interpretation dieser Situationen.

Sind Lehrkräfte in der Lage, bei der Wahrnehmung von Unterricht auf ein breites Spektrum pädagogisch-psychologischen Wissens zurückzugreifen, es mit dem theoretischen Handlungswissen zu verknüpfen und die Folgen für den weiteren Verlauf abzuschätzen, können sie Prognosen über weitere Verläufe des Unterrichts stellen (Seidel et al. 2010). Lehrkräfte, die auf dieser Ebene agieren, können daher häufig auf der Stufe des Vorhersagens operieren (Seidel et al. 2010; Stürmer et al. 2015), indem von der identifizierten Situation auf den weiteren Verlauf des Unterrichts geschlossen wird und Handlungsalternativen für den weiteren Verlauf entwickelt werden (Seidel et al. 2010). Die Relevanz der Entwicklung von Handlungsalternativen wurde von Kersting et al. (2012) betont, die zeigten, dass ,suggestions for improvement, was statistically significantly predictive of student learning" (S. 572). Bevor Handlungsalternativen generiert werden können, müssen Ereignisse zunächst erkannt und dann bewertet werden (Schwindt 2008). Der Aufbau und die Förderung dieser Kompetenzkonstrukte ist dabei maßgeblich von den zur Verfügung gestellten Lerngelegenheiten und deren Nutzung abhängig (Kunina-Habenicht et al. 2013).

\subsection{Förderung von PWKF durch videobasierte Lerngelegenheiten}

Beispiele für den gezielten Einsatz eigener und fremder Videos in der Lehrerbildung sind die von van Es und Sherin $(2008,2009)$ entwickelten Videoclubs. Im Rahmen einer Prä-Post-Erhebung durch ,Noticing Interviews', in denen die Teilnehmer zu den zuvor gezeigten Videosequenzen befragt wurden, konnten sie nachweisen, dass sich Videoclubs positiv auf die thematische Fokussierung in bestimmten Unterrichtssituationen auswirken und sich sowohl noticing als auch knowledge-based reasoning im Verlauf der Teilnahme verbesserten (Sherin und van Es 2009). Auch in anderen Studien zeigte sich, dass sich die PWKF durch die Analyse von eigenen und fremden Unterrichtsvideos in der universitären Lehramtsausbildung fördern lässt (Gold et al. 2013, 2017; Hellermann et al. 2015; Kramer et al. 2017). In einer aktuellen Studie (Weber et al. 2018) wurde die PWKF von Studierenden im vierten Bachelorsemester 
im Rahmen einer Prä-Post-Interventionsstudie untersucht. Die Auswertung der standardisierten Items des Tests zur Erfassung der PWKF (Gold und Holodynski 2017) zeigte, dass eine konventionelle Praktikumsbegleitung (KG) ohne eigene Videos die PWKF nicht signifikant fördert (KG: $d=0,20$; n. s.). Im Vergleich dazu verbesserten die Studierenden, die video- und onlinebasiertes Peer- und Expertenfeedback $(\mathrm{IG})^{1}$ erhielten, ihre PWKF signifikant (IG: $\left.d=1,10 ; p=<0,001\right)$. Hinsichtlich der verschiedenen Facetten von Klassenführung verbesserten sich die Studierenden der IG bei allen drei Facetten signifikant. Es zeigte sich, dass sich die IG bei den Facetten Monitoring (IG: $d=1,08 ; p=<0,01 ; \mathrm{KG}: d=0,15$; n. s.) und Strukturierung (IG: $d=1,00 ; p=<0,001 ; \mathrm{KG}: d=0,13$; n. s.) signifikant gegenüber der KG verbesserte. Bei Regeln und Routinen zeigte sich hingegen kein signifikanter Unterschied.

\subsection{Fragestellungen}

Bisherige Studien zur Förderung der PWKF im Studium konnten zeigen, dass diese durch die Analyse fremder und eigener Videos gefördert werden kann. Aktuelle standardisierte Verfahren können jedoch bestimmte Aspekte der professionellen Wahrnehmung vermutlich nicht komplett erfassen. Um auch Aussagen über die Förderung der selektiven Wahrnehmung und der Subprozesse Bewerten und Handlungsalternativen vorlegen sowie der Integration von Einzelereignissen in übergeordnete Fachkonzepte (Klassifikation des deklarativen Wissens) treffen zu können, erfolgt in der vorliegenden Studie eine quantitativ-inhaltsanalytische Analyse von offenen Antwortformaten. Folgende Fragestellungen lassen sich ableiten:

1 Verbessern die Studierenden der IG ihr Wissen im Sinne der Klassifikation deklarativer Wissensbestände zum Thema Klassenführung stärker als die Studierenden der $\mathrm{KG}$ ?

2 Ändert sich die selektive Wahrnehmung von Klassenführung der Studierenden der IG stärker als in der KG?

3 Haben die Studierenden der IG einen anderen Umgang mit Wertung als die Studierenden der KG?

4 Generieren die Studierenden der IG mehr Handlungsalternativen in Bezug auf Monitoring, prozessuale Strukturierung und Regeln und Routinen als die Studierenden der KG?

\footnotetext{
${ }^{1}$ Eine zweite Interventionsgruppe $(n=16)$ erhielt ausschließlich Peerfeedback und verbesserte sich nicht signifikant gegenüber der KG. Die Studierenden dieser IG wurden daher in der vorliegenden Studie nicht berücksichtigt.
} 


\section{Methode}

\subsection{Interventionsdesign}

Die Intervention ${ }^{2}$ fand im Rahmen einer quasi-experimentellen Prä-Poststudie im vierten Bachelorsemester statt. Während der Vorlesungszeit besuchten alle Lehramtsstudierenden eine Vorlesung zu Didaktik und Methodik sowie ein begleitendes Seminar, in welchem unter anderem Grundlagen der Klassenführung thematisiert wurden. Im Anschluss an die Vorlesungszeit absolvierten die Studierenden ein vierwöchiges Unterrichtspraktikum, bei welchem der Fokus auf Klassenführung lag und in welchem sie mindestens vier Unterrichtsstunden selbstverantwortlich planen und durchführen sollten. Die Studierenden wurden während des Praktikums in Kleingruppen von 10-13 Studierenden von Dozierenden der Universität betreut. Studierende der KG erhielten während des vierwöchigen Zeitraumes zwei Besuche von ihren Dozierenden sowie ihren Team- und Tandempartnerinnen und -partnern. Nach den praktischen Unterrichtsversuchen erfolgte eine face-to-face Reflexion mit dem Fokus auf Klassenführung und Feedback von ihren Peers und den Dozierenden.

In der IG wurden die Studierenden von ihren Teampartnern während eigener Unterrichtsstunden gefilmt, so dass alle Studierenden über zwei Aufnahmen eigenen Unterrichts verfügten. Schriftliche Einverständniserklärungen lagen dabei sowohl von den Studierenden als auch von den Schulleiterinnen und Schulleitern der entsprechenden Schulen vor. Zudem wurde in den Klassen in denen gefilmt wurde, von allen Schülerinnen und Schülern die schriftliche Einverständniserklärung der Eltern eingeholt. Die Studierenden wählten jeweils eine 5- bis 10-minütige Sequenz aus ihren Unterrichtsstunden aus und luden diese auf der Online-Lern-Plattform ,Moodle hoch, wo die Videosequenzen kommentiert werden konnten. Bei der Auswahl der Videoclips war darauf zu achten, dass sie eine Instruktions- und die anschließende

1.

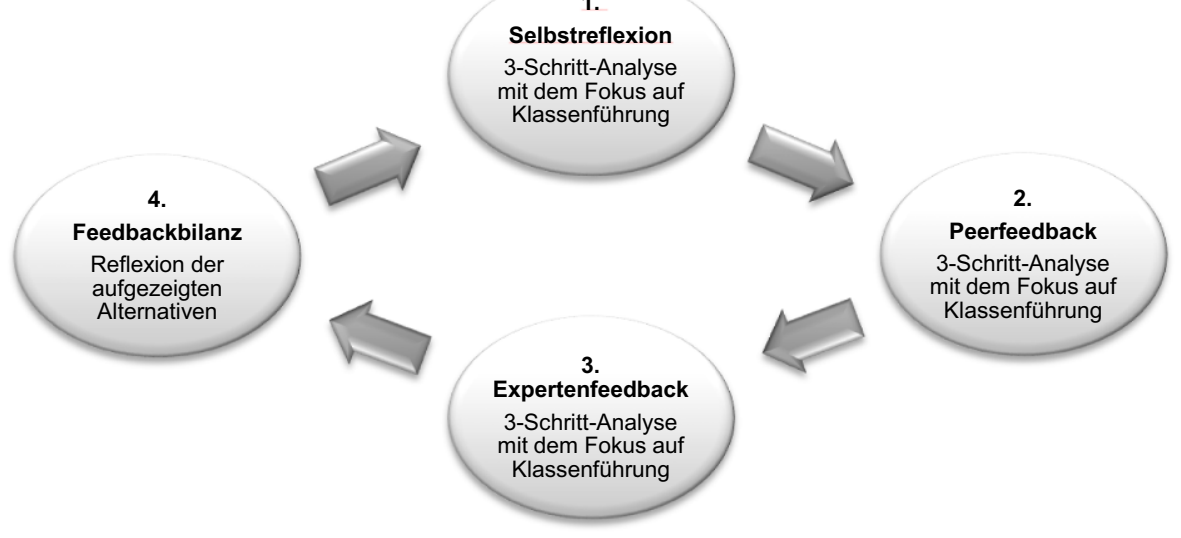

Abb. 1 Feedbackzirkel. (Nach Kleinknecht und Gröschner 2016; Weber et al. 2018)

${ }^{2}$ Für ausführlichere Informationen zum Interventionsdesign siehe Weber et al. (2018). 
Übergangsphase zu einer anderen Lernaktivität aufwiesen. Darüber hinaus sollte jede Sequenz mindestens ein kritisches Element zur Klassenführung enthalten, welches während des Unterrichtens stattfand. Die Studierenden der IG erhielten ebenfalls ein face-to-face Reflexions- und Feedbackgespräch. Als Ersatz für das zweite faceto-face Gespräch erfolgte die Reflexion des eigenen Unterrichts anhand der zwei eigenen Videosequenzen und die Studierenden erhielten anschließend online- und videobasiertes Peer- und Expertenfeedback. Sowohl beim face-to-face Feedbackgespräch als auch in den online-Foren sollten die Studierenden einen strukturierten Reflexions- und Feedbackprozess durchlaufen (s. Abb. 1). Dieser bestand aus einem Dreischritt aus Beschreiben von spezifischen Unterrichtsituationen, Bewerten/ Interpretieren dieser Unterrichtssituationen und Entwickeln von Handlungsalternativen (Seidel et al. 2011; Seidel und Stürmer 2014; Kleinknecht und Gröschner 2016; Weber et al. 2018). Durch die Verwendung des Dreischritts sollte eine rein beschreibende Selbstreflexion sowie ein oberflächliches Feedback vermieden werden. Zudem wurde das Entwickeln von Handlungsalternativen durch den Dreischritt gezielt geschult. Bei der Feedbackbilanz sollten die Studierenden anhand der eigenen Reflexion und der erhaltenen Feedbacks die gewonnen Erkenntnisse mit den bestehenden Wissensbeständen abgleichen um diese ggf. zu erweitern und anschlieBend in neuen Reflexions- und Feedbackprozessen erneut zur Disposition zu stellen (siehe auch Weber et al. 2018).

\subsection{Erhebungsinstrument}

Die PWKF wurde mit einem videobasierten Test von Gold und Holodynski (2017) gemessen, der von den Studierenden online über Unipark (Globalpark 2011) bearbeitet wurde. Der Test besteht aus kurzen Videoclips von Unterrichtssituationen, die die Probanden in geschlossenen und offenen Items bearbeiten mussten. Die geschlossenen Items ließen sich mittels standardisierter Ratingskalen bewerten, während die offenen Items anhand zweier, auf das Video bezogener, Fragen zu beantworten waren.

1 Welche klassenführungsspezifischen Maßnahmen ergreift die Lehrerin in diesem Ausschnitt und wie wirken diese Maßnahmen auf das Schülerverhalten? Bitte begründen Sie.

2 Hätte die Lehrerin weitere andere (ggf. effektivere) klassenführungsspezifische Maßnahmen ergreifen sollen? Bitte begründen Sie.

Beide Fragen verweisen auf die klassenführungsspezifischen Maßnahmen, die die Lehrerin in dieser Szene ergreift. Bevor die Studierenden die Maßnahmen wissensgesteuert verarbeiten können, müssen sie zunächst (selektiv) wahrnehmen, welche Maßnahmen die Lehrerin ergreift. Die Antworten der Studierenden im Prä- und die Posttest bilden entsprechend die Datengrundlage der vorliegenden Studie. Die Antworten zu beiden Fragen wurden dabei einzeln kodiert und anschließend zusammengeführt, indem das gewichtete arithmetische Mittel gebildet wurde. Die gewichteten 
arithmetischen Mittelwerte sagen dabei aus, auf welchem Niveau der Kategoriensysteme (siehe Abschn. 2.4) die Probanden im Prä- und Posttest agieren.

\subsection{Unterteilung in Sinnkategorien und Sinneinheiten}

Das Video, auf welches sich beide Fragen beziehen, zeigt einen Ausschnitt aus einer Unterrichtsstunde zum Schwimmen und Sinken. Es zeigt den Übergang von einer Gruppenarbeit in eine Präsentationsphase. Um die Fragen der beiden offenen Items analysieren und auswerten zu können, wurde die Videosequenz zunächst in einzelne Sinnkategorien (SK) und -einheiten (SE) gegliedert. Jede SK bildet dabei einen Aspekt des Übergangsmanagements nach Thiel et al. (2012) - Beendigung des alten Handlungsvektors, Etablierung des neuen Handlungsvektors und Stabilisierung des neuen Handlungsvektors - ab. Anhand der für Experten typischen Übergangsverläufe erfolgt eine Modellierung der relevanten Phasen in einzelne Abschnitte, die dem Verlauf der Unterrichtssequenz zugeordnet werden. Zusätzlich wurden diese übergeordneten SK in SE aufgegliedert. Jede SE bildet einen Handlungsaspekt der Unterrichtssequenz ab (z. B. Lehrkraft schließt alte Unterrichtsaktivität ab und leitet über zu einer Neuen). Diese zusätzliche Ausdifferenzierung soll dazu dienen, den Umgang mit konkurrierenden Handlungsvektoren, über die PWKF hinsichtlich der Aspekte Monitoring, prozessuale Strukturierung sowie Regeln und Routinen, detaillierter abbilden zu können. Es lassen sich drei SK voneinander unterscheiden, denen sich insgesamt neun SE zuordnen lassen (siehe Tab. 1). Für die Auswertung der Testergebnisse wurde nicht nur das Unterrichtsvideo in Subsequenzen unterteilt, sondern auch die Antworten der Studierenden in Sinneinheiten aufgegliedert, in denen jeweils alle Aussagen zusammengefasst wurden, die sich inhaltlich auf eine der SK bzw. SE aus dem Video bezogen. Nach der erfolgten Zuordnung wurden die zugeordneten SE aus den Testantworten innerhalb der SK bzw. SE auf die unterschiedlichen Teilkompetenzen und Verlaufsphasen hin analysiert.

\subsection{Kodierung der Daten}

\subsubsection{Kodierung der Klassifikation der deklarativen Wissensbestände}

Um Rückschlüsse auf die Veränderung der Klassifikation der deklarativen Wissensbestände zum Thema Klassenführung ziehen zu können, wurde in Anlehnung an

Tab. 1 Schematische Abbildung eines Ausschnitts aus der Vergleichsfolie zu den einzelnen Verlaufsphasen

\begin{tabular}{lllll}
\hline SK & SE & $\begin{array}{l}\text { Verlaufsphase } \\
\text { (Zeit) }\end{array}$ & Handlungsvektor & Beschreibung \\
\hline 1 & 2 & Beendigungsprozess & Beendigung & Die Lehrkraft steht von ihrem Platz auf und \\
& der Aktivitäts- & des alten & schlägt am äußeren Rand des Klassenraumes \\
& struktur & Handlungs- & auf den Gong. Sie fragt, welche Gruppe anfan- \\
& $(00: 14-00: 38)$ & vektors & gen möchte, vorzustellen \\
\hline
\end{tabular}

Insgesamt wurden drei Sinnkategorien (SK) unterschieden (Beendigung des alten Handlungsvektors, Etablierung des neuen Handlungsvektors und Stabilisierung des neuen Handlungsvektors) denen jeweils drei Sinneinheiten (SE) zugeordnet wurden 
Schwindt (2008) erfasst, inwieweit die Probanden in der Lage sind, ihre Antworten in übergeordnete Konzepte einzubetten und diese ggf. mit den zugehörigen Fachbegriffen zu benennen. Als Analyseeinheit wurden die einzelnen identifizierten SE der Probandenantworten für jede Frage herangezogen und individuell, anhand von drei Ausprägungsstufen, kodiert: $0=$ Beschreibung von Einzelereignissen; 1 = Alltagssprachliche Integration von Einzelereignissen in übergeordnete Konzepte, $2=$ Einzelereignisse werden unter der Verwendung der richtigen Fachbegriffe zusammengefasst und eingeordnet. Als finaler Wert der Kodierung wurde das gewichtete arithmetische Mittel für jeden Probanden und jede Frage errechnet.

\subsubsection{Kodierung der selektiven Wahrnehmung von Handlungsübergängen}

Der Fokus in der Kodierung der selektiven Wahrnehmung von Handlungsübergängen lag darauf, wie viele unterschiedliche Verlaufsphasen Novizinnen und Novizen wahrnehmen. Für die Ermittlung der selektiven Wahrnehmung wurde eine Vergleichsfolie zu den einzelnen Verlaufsphasen angefertigt (vgl. Thiel et al. 2012; vgl. Tab. 1). Zu deren Erstellung wurden die abstrahierten Ergebnisse von Thiel et al. (2012) durch die spezifischen Ergebnisse einer Expertenlösung von Gold und Holodynski (2017) ergänzt und auf die einzelnen, aus der Sequenzanalyse des Unterrichtsmitschnitts gewonnenen, SK und SE bezogen. Zunächst wurde rein quantitativ überprüft, wie viele der identifizierten SE sich auf die übergeordneten SK bezogen, um zu erfassen, mit welcher Intensität die einzelnen Übergangsphasen wahrgenommen wurden und wie sich die Wahrnehmung veränderte. Für die weitere Auswertung wurden für die Sinnkategorien pro Gruppe und Messzeitpunkt der Mittelwert gebildet. Der Mittelwert wurde dabei über die Anzahl von wahrgenommenen SE innerhalb der SK berechnet.

\subsubsection{Kodierung der wissensgesteuerten Verarbeitung von Unterricht}

Die identifizierten SE der Studierenden wurden bezüglich der klassenführungsrelevanten Maßnahmen der Lehrerin innerhalb der einzelnen SE der Unterrichtssequenz untersucht. Die Überprüfung erfolgte anhand einer Vergleichsfolie und eines Kodiermanuals, das auf der Basis der Schwerpunkte aus dem Begleitseminar - Monitoring, prozessuale Strukturierung und Regeln und Routinen - sowie der Ergebnisse von Thiel et al. (2012) und der Expertenlösung erstellt wurde. Um die wissensgesteuerte Verarbeitung von Unterricht zu kodieren wurde auf ein Kategoriensystem von Schwindt (2008) zurückgegriffen. Dabei wurden die Facetten Monitoring, prozessuale Strukturierung und Regeln und Routinen einzeln kodiert. Das Kodierverfahren erfolgte, indem die in einzelne SE segmentierten Antworten der Probanden den jeweils zugehörigen SE der Videosequenz zugeordnet wurden. Daraufhin wurden die SE der Probandenantwort auf die einzelnen Dimensionen der Klassenführung hin analysiert. Für die Kodierung der Antworten wurde auf die Subprozesse der professionellen Wahrnehmung: Beschreiben, Erklären, Interpretieren, Vorhersagen und Handlungsalternativen vorlegen zurückgegriffen $(0=$ unzureichende/falsche Antwort; 1 = Beschreibung; 2 =Erklärung/Interpretation; $3=$ Beschreibung, Erklärung/Interpretation und Vorhersagen/Handlungsalternativen vorlegen). Die Kodie- 
rungen für die einzelnen SE wurden anschließend zusammengefasst, indem für jede Dimension und Gruppe das gewichtete arithmetische Mittel gebildet wurde.

\subsubsection{Kodierung Umgang mit Wertung}

Eine Vielzahl von Studien orientiert sich an den drei Facetten Beschreiben, Erklären und Bewerten (u.a. Seidel und Prenzel 2007; Schwindt 2008). Um den Umgang mit Bewertungen differenzierter zu betrachten und eine Vergleichbarkeit mit den Ergebnissen anderer Studien zu ermöglichen, wurde der ,Umgang mit Wertung' in Anlehnung an Schwindt (2008) und Kleinknecht und Schneider (2013) kodiert: $0=$ Keine positiven/kritischen Ereignisse erkannt; $1=$ Beschreibung positiver/ kritischer Ereignisse; 2 =Bewertung positiver/kritischer Ereignisse; $3=$ Beschreibung und Bewertung positiver/kritischer Ereignisse und Aufzeigen von Konsequenzen; 4 = Beschreibung und Bewertung positiver/kritischer Ereignisse, Aufzeigen von Konsequenzen und begründeter Handlungsalternativen. Als Wertung wurden nur Antworten berücksichtigt, die sich explizit auf beobachtete Lehrerhandlungen bezogen. Verallgemeinerungen wurden nicht berücksichtigt. Mit dieser Herangehensweise wurden alle SE der Probandenantworten kodiert. Im Anschluss wurde für jede Teilnehmerin und jeden Teilnehmer das gewichtete arithmetische Mittel gebildet und mit diesem Wert als Indikator für die Qualität der Bewertungen weitergearbeitet.

\subsection{Aggregation der Daten und statistische Analysen}

Es wurden die verschiedenen Analysebereiche für jeden Probanden gemäß des Kodiermanuals ausgewertet. In die Auswertung wurden nur jene SE des Videos einbezogen, denen sich eine SE aus der jeweiligen Antwort zuordnen ließ. Die Anzahl der zugeordneten SE wurden nicht berücksichtigt. Unzureichend beantwortete Teile wurden mit einer 0 kodiert und somit in der Gesamtbewertung berücksichtigt. Um eine möglichst objektive Kodierung zu gewährleisten, wurden die Antworten unabhängig von Messzeitpunkt und Gruppenzugehörigkeit kodiert. Erst im Nachhinein wurden die Prä- und Posttestungen der einzelnen Teilnehmer wieder zusammengeführt und der jeweiligen Gruppe zugeordnet. Neben der Darstellung der prozentuellen Häufigkeiten der kodierten Antworten sind die Ergebnisse als Median berichtet. Zur Analyse der Veränderung über die Zeit wurden für die selektive Wahrnehmung $t$ tests für verbundene Stichproben berechnet sowie Effektstärken bei Messwiederholung mit einer Gruppe ( $d$ zwischen 0,2 und 0,5=kleiner Effekt, $d$ zwischen 0,5 und $0,8=$ mittlerer Effekt und $d>0,8=$ starker Effekt, Cohen 1988). Da nicht gewährleistet ist, dass die kodierten Antworten zur wissensgesteuerten Verarbeitung von Unterricht äquidistante Abstände anzeigen, wurden die vorliegenden Daten mittels nicht parametrischer Verfahren analysiert. Hierzu wurde die Differenz zwischen präund posttest berechnet (Differenzvariable) und der Mann-Whitney- $U$-Test durchgeführt, mit der Differenzvariable als abhängige Variable und Gruppenzugehörigkeit als unabhängige Variable. Die Effektstärken wurden mit dem Korrelationskoeffizienten $(r)$ von Pearson berechnet und nach Cohen (1988) interpretiert ( $r$ zwischen 0,1-0,3=kleiner Effekt; 0,3-0,5=mittlerer Effekt; $r \geq 0,5=$ starker Effekt). 
Tab. 2 Prozentuale Übereinstimmung und Cohens Kappa

\begin{tabular}{lll}
\hline Bereich & Prozentuale Übereinstimmung & Cohens Kappa \\
\hline Selektive Wahrnehmung & & \\
Phasenübergang - Beendigung & 88,9 & 0,83 \\
Phasenübergang - Etablierung & 88,9 & 0,86 \\
Phasenübergang - Stabilisierung & 77,8 & 0,69 \\
Phasenübergang - Gesamt & 85,2 & 0,81 \\
Wissensgesteuerte Verarbeitung von Unterricht & & \\
Klassenführung - Monitoring & 84,4 & 0,75 \\
Klassenführung - Prozessuale Strukturierung & 86,4 & 0,79 \\
Klassenführung - Regeln und Routinen & 88,0 & 0,81 \\
Umgang mit Wertung & 87,0 & 0,82 \\
Klassifikation der deklarativen Wissensbestände & & \\
Klassifikation & 91,0 & 0,79 \\
\hline
\end{tabular}

Prozentuale Übereinstimmung $>85 \%$ sowie Cohens Kappa $>0,7=$ gute Interraterübereinstimmung Cohens Kappa sollte mindestens über >0,60 liegen (Landis und Koch 1977)

\subsection{Interraterreliabilität}

Die Reliabilität der einzelnen Kategorien wurde anhand zufällig gewählter Testantworten von neun Teilnehmern ( $n=9 ; 18$ Fragen) überprüft (Schwindt 2008). Zwei unabhängige Kodierer erprobten das Kodiermanual, indem sie jeweils beide Fragen zum Videoclip kodierten, wobei die für die Doppelkodierung genutzten Datensätze anschließend nicht in die finale Auswertung eingingen. Im Anschluss wurde die Interraterübereinstimmung errechnet. Nach Schwindt (2008) kann man von einer guten Übereinstimmung ausgehen, wenn eine direkte prozentuale Übereinstimmung von über $85 \%$ sowie ein Cohens Kappa von über 0,70 vorliegt (vgl. auch Wirtz und Caspar 2002; nach Landis und Koch 1977 sollte Cohens Kappa mindestens über $>0,60$ liegen). Obwohl die Werte bei Stabilisierung etwas niedriger ausfallen, befinden sich diese noch im akzeptablen Bereich. Die Kodiersysteme erwiesen sich entsprechend als reliabel (vgl. Tab. 2).

\subsection{Stichprobe der vorliegenden Studie}

Um die forschungsethischen Prinzipien der freiwilligen Teilnahme und der informierten Einwilligung zu gewährleisten, wurden alle Studierenden im Rahmen der praktikumsvorbereitenden Vorlesung über die Möglichkeit informiert, sich während des Praktikums zu filmen. Es wurden nur Studierende gefilmt, die zuvor ihr schriftliches Einverständnis gegeben hatten. Das schriftliche Einverständnis konnte zudem zu jedem Zeitpunkt zurückgezogen werden. Entsprechend war keine Randomisierung der Studierenden zur IG und KG möglich. Die IG umfasste 26 Studierende ( $96 \%$ weiblich, $M_{\text {Alter }}=22,64 ; S D_{\text {Alter }}=3,97$ ). Die KG bestand insgesamt aus 69 Studierenden, von denen $n=26$ Studierende (96\% weiblich, $\left.M_{\text {Alter }}=23,62 ; S D_{\text {Alter }}=4,37\right)$ zufällig für die vorliegenden Analysen ausgewählt wurden. Im Prätest unterschieden sich die beiden Gruppen nur in einem Analy- 
sebereich signifikant voneinander, wobei die KG signifikant besser im Bereich der PW von Monitoring abschnitt: selektive Wahrnehmung - Beendigung des alten Handlungsvektors: $M_{I G}=1,64 ; S D=0,49 ; M_{K G}=1,61 ; S D=0,77 ; p=0,89 ;$ selektive Wahrnehmung - Etablierung des neuen Handlungsvektors: $M_{I G}=1,58 ; S D=0,58$; $M_{K G}=1,75 ; S D=0,74 ; p=0,38 ;$ selektive Wahrnehmung - Stabilisierung des neuen Handlungsvektors: $M_{I G}=1,89 ; S D=0,98 ; M_{K G}=1,79 ; S D=0,86 ; p=0,73 ;$ Monitoring: $M d_{I G}=1,34 ; M d_{K G}=1,71 ; U=219,00 ; \mathrm{Z}=-2,041 ; p<0,05 ;$ prozessuale Strukturierung: $M d_{I G}=1,67 ; M d_{K G}=1,67 ; U=307,50 ; Z=-0,098 ; p=0,92 ;$ Regeln und Routinen: $M d_{I G}=2,0 ; M d_{K G}=1,5 ; U=178,50 ; \mathrm{Z}=-1,338 ; p=0,181 ;$ Umgang mit Wertung: $M d_{I G}=1,79 ; M d_{K G}=1,85 ; U=304,50 ; Z=-0,614 ; p=0,54$ und Klassifikation: $M d_{I G}=1,0 ; M d_{K G}=1,0 ; U=255,00 ; \mathrm{Z}=-1,600 ; p=0,110$.

\section{Ergebnisse}

\subsection{Veränderung der Klassifikation der deklarativen Wissensbestände}

Bezogen auf die Fähigkeit Einzelereignisse unter Verwendung der richtigen Fachbegriffe zusammenzufassen und einzuordnen, lassen sich im Zuge der Intervention für beide Gruppen Verbesserungen feststellen. Während im Prätest sowohl in der IG als auch in der KG nur 3,8\% der Ereignisse unter Verwendung der richtigen Fachbe-

Tab. 3 Mittelwerte $(M)$, Standardabweichungen $(S D)$ und Effektstärke (Cohens $d$ ) der Veränderungen zwischen Prä- und Posttest für die Selektive Wahrnehmung

\begin{tabular}{|c|c|c|c|c|c|c|c|c|c|}
\hline & \multicolumn{2}{|c|}{ Prätest } & \multicolumn{2}{|c|}{ Posttest } & \multirow[b]{2}{*}{$\Delta$} & \multirow[b]{2}{*}{$t$} & \multirow[b]{2}{*}{$D f$} & \multirow[b]{2}{*}{$p$} & \multirow[b]{2}{*}{$d$} \\
\hline & $M$ & $S D$ & $M$ & $S D$ & & & & & \\
\hline \multicolumn{10}{|c|}{ Selektive Wahrnehmung } \\
\hline \multicolumn{10}{|c|}{ Beendigung alter Handlungsvektor } \\
\hline $\begin{array}{l}\text { Kontrollgruppe } \\
(\mathrm{KG})\end{array}$ & 1,67 & 0,78 & 1,50 & 0,47 & $-0,17$ & $-0,85$ & 20 & 0,406 & $-0,15$ \\
\hline $\begin{array}{l}\text { Interventionsgruppe } \\
\text { (IG) }\end{array}$ & 1,62 & 0,50 & 1,14 & 0,32 & $-0,48$ & $-3,91$ & 20 & 0,001 & $-0,72$ \\
\hline \multicolumn{10}{|c|}{ Etablierung neuer Handlungsvektor } \\
\hline $\begin{array}{l}\text { Kontrollgruppe } \\
(\mathrm{KG})\end{array}$ & 1,74 & 0,75 & 1,70 & 0,63 & $-0,04$ & $-0,20$ & 24 & 0,841 & $-0,04$ \\
\hline $\begin{array}{l}\text { Interventionsgruppe } \\
\text { (IG) }\end{array}$ & 1,58 & 0,58 & 1,69 & 0,57 & 0,10 & 0,77 & 23 & 0,447 & 0,16 \\
\hline \multicolumn{10}{|c|}{ Stabilisierung neuer Handlungsvektor } \\
\hline $\begin{array}{l}\text { Kontrollgruppe } \\
\text { (KG) }\end{array}$ & 1,78 & 0,77 & 1,53 & 0,87 & $-0,25$ & $-1,34$ & 17 & 0,197 & $-0,34$ \\
\hline $\begin{array}{l}\text { Interventionsgruppe } \\
\text { (IG) }\end{array}$ & 1,80 & 0,89 & 1,58 & 0,86 & $-0,23$ & $-0,75$ & 19 & 0,463 & $-0,17$ \\
\hline \multicolumn{10}{|c|}{ Selektive Wahrnehmung gesamt } \\
\hline $\begin{array}{l}\text { Kontrollgruppe } \\
\text { (KG) }\end{array}$ & 1,73 & 0,56 & 1,57 & 0,38 & $-0,16$ & $-1,18$ & 24 & 0,249 & $-0,20$ \\
\hline $\begin{array}{l}\text { Interventionsgruppe } \\
\text { (IG) }\end{array}$ & 1,71 & 0,48 & 1,51 & 0,43 & $-0,20$ & $-1,83$ & 25 & 0,079 & $-0,35$ \\
\hline
\end{tabular}


Tab. 4 Prozentuale Verteilung auf die verschiedenen Kodierungen sowie Median im Prä- und Posttest

\begin{tabular}{|c|c|c|c|c|}
\hline & \multicolumn{2}{|c|}{ Kontrollgruppe } & \multicolumn{2}{|c|}{ Interventionsgruppe } \\
\hline & Prätest & Posttest & Prätest & Posttest \\
\hline \multicolumn{5}{|l|}{ Klassifikation deklarativer Wissensbestände } \\
\hline $0=$ Beschreibung von Einzelereignissen & 15,4 & 3,8 & 26,9 & 0,0 \\
\hline $\begin{array}{l}1=\text { Alltagssprachliche Integration von Ereignissen } \\
\text { in übergeordnete Konzepte }\end{array}$ & 80,8 & 73,1 & 69,1 & 53,8 \\
\hline $\begin{array}{l}2=\text { Einordnung von Ereignissen unter Verwen- } \\
\text { dung der richtigen Fachbegriffe }\end{array}$ & 3,8 & 23,1 & 3,8 & 46,2 \\
\hline Median & 1,0 & 1,25 & 1,0 & 1,45 \\
\hline \multicolumn{5}{|l|}{ Wissensgesteuerte Verarbeitung von Unterricht } \\
\hline \multicolumn{5}{|l|}{ Monitoring } \\
\hline $0=$ unzureichende/falsche Antwort & 0,0 & 0,0 & 4,0 & 3,8 \\
\hline $1=$ Beschreibung & 30,8 & 26,9 & 48,0 & 0,0 \\
\hline $2=$ Erklärung/Interpretation & 61,5 & 69,3 & 48,0 & 61,6 \\
\hline $\begin{array}{l}3=\text { Beschreibung, Erklärung/Interpretation und } \\
\text { Vorhersagen/Handlungsalternativen vorlegen }\end{array}$ & 7,7 & 3,8 & 0,0 & 34,5 \\
\hline Median & 1,71 & 1,58 & 1,34 & 2,0 \\
\hline \multicolumn{5}{|l|}{ Prozessuale Strukturierung } \\
\hline $0=$ unzureichende/falsche Antwort & 4,0 & 0,0 & 0,0 & 0,0 \\
\hline $1=$ Beschreibung & 20,00 & 22,7 & 32,0 & 12,0 \\
\hline $2=$ Erklärung/Interpretation & 64,00 & 72,8 & 64,0 & 60,0 \\
\hline $\begin{array}{l}3=\text { Beschreibung, Erklärung/Interpretation und } \\
\text { Vorhersagen/Handlungsalternativen vorlegen }\end{array}$ & 12,00 & 4,5 & 4,0 & 28,0 \\
\hline Median & 1,67 & 1,71 & 1,67 & 2,00 \\
\hline \multicolumn{5}{|l|}{ Regeln und Routinen } \\
\hline $0=$ unzureichende/falsche Antwort & 0,0 & 0,0 & 0,0 & 3,8 \\
\hline $1=$ Beschreibung & 36,4 & 36,0 & 28,6 & 7,7 \\
\hline $2=$ Erklärung/Interpretation & 50,0 & 52,0 & 52,4 & 46,2 \\
\hline $\begin{array}{l}3=\text { Beschreibung, Erklärung/Interpretation und } \\
\text { Vorhersagen/Handlungsalternativen vorlegen }\end{array}$ & 13,6 & 12,0 & 19,1 & 42,3 \\
\hline Median & 1,5 & 1,67 & 2,0 & 2,0 \\
\hline \multicolumn{5}{|l|}{ Umgang mit Wertung } \\
\hline $0=$ Keine positiven $/$ kritischen Ereignisse erkannt & 3,8 & 0,00 & 3,8 & 0,00 \\
\hline $1=$ Beschreibung positiver/kritischer Ereignisse & 19,3 & 11,5 & 27,0 & 0,00 \\
\hline $2=$ Bewertung positiver $/$ kritischer Ereignisse & 53,8 & 50,0 & 57,7 & 42,3 \\
\hline $\begin{array}{l}3=\text { Beschreibung und Bewertung und Aufzeigen } \\
\text { von Konsequenzen }\end{array}$ & 23,1 & 38,5 & 11,5 & 46,2 \\
\hline $\begin{array}{l}4=\text { Beschreibung und Bewertung, Aufzeigen von } \\
\text { Konsequenzen und begründeter Handlungsalterna- } \\
\text { tiven }\end{array}$ & 0,0 & 0,0 & 0,0 & 11,5 \\
\hline Median & 1,85 & 2,19 & 1,79 & 2,58 \\
\hline
\end{tabular}


griffe eingeordnet werden, sind es im Posttest $23,1 \%$ bei der $\mathrm{KG}$ und $46,2 \%$ bei der IG (siehe Tab. 4). Die Ergebnisse des $U$-Test nach Mann und Whitney zeigen, dass sich die IG signifikant mehr verbessert als die KG, $U=198,00 ; Z=-2,564 ; p<0,05$ mit einer mittleren Effektstärke von $r=0,36$.

\subsection{Veränderung der selektiven Wahrnehmung}

Es zeigt sich, dass sich die Anzahl der identifizierten SE in beiden Gruppen in fast allen Bereichen reduziert und im Posttest eine geringere Anzahl relevanter SE identifiziert wurde, als dies im Prätest der Fall war. Jedoch geht aus Tab. 3 hervor, dass diese Unterschiede in der selektiven Wahrnehmung lediglich in der IG für die Wahrnehmung der Beendigung signifikant sind. In der KG zeigt sich kein signifikanter Rückgang (siehe Tab. 3).

\subsection{Veränderung des Umgangs mit Wertung}

Für den Umgang mit Wertung zeigt sich, dass die meisten Studierenden bereits im Prätest Einzelereignisse bewerten, jedoch kaum Konsequenzen aufzeigen und begründete Handlungsalternativen generieren (siehe Tab. 4). Im Posttest werden 38,5\% (KG) und 46,2\% (IG) der Ereignisse beschrieben, bewertet und Konsequenzen aufgezeigt. In der KG werden jedoch weiterhin keine zusätzlichen Handlungsalternativen generiert, während in der IG 11,5\% der Ereignisse nach Schwindt (2008) konstruktiv bewertet und diskutiert werden (höchste Kodierung, siehe Tab. 4). Der $U$-Test nach Mann und Whitney zeigt dabei, dass sich die IG beim Umgang mit Wertung signifikant mehr verbessert als die $\mathrm{KG}, U=212,00 ; \mathrm{Z}=-2,306 ; p<0,05$ mit einer mittleren Effektstärke von $r=0,32$.

\subsection{Veränderung im Generieren von Handlungsalternativen}

Bei der Analyse der Posttest-Daten zeigt sich in allen Bereichen, dass in der IG sehr viel häufiger die höchstmögliche Kodierung 3 (Beschreibung, Interpretation/ Erklärung und Vorhersagen/Handlungsalternativen vorlegen) vergeben wurde (siehe Tab. 4). Bei Monitoring wurde die Kodierung 3 in 34,5\% aller Fälle vergeben, während in der KG nur 3,8\% der Fälle mit 3 kodiert wurden. Im Bereich der prozessualen Strukturierung lag der prozentuelle Anteil bei der IG bei $28 \%$, während diese in der $\mathrm{KG}$ nur in 4,5\% aller Fälle Anwendung fand. Bezüglich Regeln und Routinen zeigt sich, dass die Kodierung 3 in beiden Gruppen prozentual gesehen häufiger vergeben wird als im Bereich des Monitorings oder der prozessualen Strukturierung, wobei der Wert in der IG mit 42,3\% auch hier höher ausfällt als für die KG mit $12 \%$. Die Ergebnisse des $U$-Test nach Mann und Whitney zeigen, dass sich die IG im Bereich des Monitoring signifikant stärker verbesserte als die $\mathrm{KG}, U=141,00 ; \mathrm{Z}=-3,482 ; p<0,001$ mit einer mittleren bis hohen Effektstärke von $r=0,48$. Bei prozessualer Strukturierung zeigt sich kein signifikanter Unterschied, $U=183,00 ; Z=-1,759 ; p=0,078$, ebenso wenig wie bei Regeln und Routinen, $U=164,00 ; Z=-1,457 ; p=0,145$. 


\section{Diskussion}

Die erste Forschungsfrage ging der Veränderung der Klassifikation der deklarativen Wissensbestände zum Thema Klassenführung nach. Da Novizen häufig Schwierigkeiten haben, ihr fachliches und pädagogisches Wissen im Prozess des Reflektierens von konkreten Unterrichtssituationen anzuwenden (Blomberg et al. 2014), wurde untersucht, inwieweit die Teilnehmer in der Lage sind, ihre Unterrichtswahrnehmungen fachsprachlich in übergeordnete Konzepte zu integrieren. Dabei erreichten beide Gruppen eine Verbesserung in dieser Kategorie. Dass sich die Ergebnisse zur Klassifikation der wahrgenommenen Ereignisse in beiden Gruppen verbesserten, spricht dafür, dass die praktikumsbegleitenden Seminare oder auch das Praktikum an sich in beiden Gruppen zur Entwicklung der Klassifikation des deklarativen Fachwissens beitrugen (Besa und Büdcher 2014). Jedoch weisen die Ergebnisse des $U$ Test nach Mann und Whitney darauf hin, dass in der IG ein höheres Maß an Wissen über die Fachkonzepte und eine größere Kompetenz in der Verwendung der richtigen Fachsprache vorhanden ist. Dies deutet darauf hin, dass die realitätsnahe Anwendung des zuvor erworbenen Wissens in der Arbeit mit Unterrichtsvideos zu einer höheren Aktivierung deklarativer Wissensbestände geführt hat, als dies in der KG der Fall war (Blomberg et al. 2011).

Hinsichtlich der zweiten Forschungsfrage war unsere Hypothese, dass die Anzahl an wahrgenommenen Sinneinheiten in der IG mehr sinkt als in der KG, was für eine stärker fokussierte Wahrnehmung sprechen würde. Im Verlauf der Ausbildung und mit wachsender Erfahrung lernen angehende Lehrkräfte unwichtige Dinge auszublenden und ihre Wahrnehmung auf relevante Aspekte zu fokussieren (van Es und Sherin 2008), die dann zunehmend elaborierter analysiert werden (Seidel et al. 2010). Die Ergebnisse der vorliegenden Studie können dies teilweise bestätigen. So sank im Prä-Postvergleich in beiden Gruppen und nahezu allen Bereichen die Anzahl der wahrgenommenen Sinneinheiten. Die Ergebnisse der IG weisen darauf hin, dass in der Posttestung bei der Wahrnehmung der Beendigung des alten Handlungsvektors mehr fokussiert wurde und die Studierenden somit einen Zuwachs in einem Bereich ihrer selektiven Wahrnehmung aufweisen, während sich in der KG keine signifikanten Veränderungen feststellen ließen. Dennoch muss bei der vorliegenden Messung der selektiven Wahrnehmung kritisch hinterfragt werden, ob eine Verringerung der Zahl der identifizierten Ereignisse von Prä- zu Posttest als Indikator für selektive Wahrnehmung gelten kann. In anderen Studien wird selektive Wahrnehmung beispielsweise durch das gezielte Stoppen von Videos gemessen (Seidel et al. 2011), durch das Kodieren von Noticing Interviews (van Es und Sherin 2008; Sherin und van Es 2009) oder durch die Verwendung von „tagging tools“ (Walkoe et al. 2019). Weiterführende Studien sollten hier anknüpfen und die Förderung selektiver Wahrnehmung durch eine videobasierte Intervention im Praktikum gezielter untersuchen, auch indem Lösungen der Studierenden mit einer Masterlösung von Expertinnen und Experten verglichen werden.

Die dritte Forschungsfrage bezog sich auf den Umgang mit Wertung bei den Studierenden der IG und der KG. Nach Schwindt (2008) ist es relevant Ereignisse nicht nur zu beurteilen sondern auch alternative Vorgehensweisen zu generieren, „um eine nachvollziehbare Einschätzung des Geschehens zu erreichen“ (S. 62). Un- 
sere Ergebnisse zeigen, dass die Studierenden der IG nach dem Praktikum deutlich mehr Ereignisse beschreiben, bewerten, Konsequenzen aufzeigen sowie begründete Handlungsalternativen formulieren als die Studierenden der KG. Eine videobasierte und strukturierte Lernumgebung scheint die konstruktive Bewertung relevanter Unterrichtsereignisse daher stärker zu fördern als eine konventionelle Praktikumsbetreuung. Diese Interpretation geht einher mit den Ergebnissen anderer Studien, die zeigen konnten, dass der Einsatz von Unterrichtsvideos im Praktikum Feedback und Analysen spezifischer und umfassender machte, während es face-to-face Nachbesprechungen von Unterrichtssituationen oftmals an Struktur fehlt (z. B. Rich und Hannafin 2008; Tripp und Rich 2012).

Mit der vierten Forschungsfrage wurde untersucht, ob die Studierenden der IG nach der Intervention mehr Handlungsalternativen in Bezug auf die verschiedenen Facetten der Klassenführung generieren als die Studierenden der KG. Bezogen auf Monitoring zeigte sich, dass sich die IG hier signifikant mehr verbesserte als die KG. Die Studierenden aus der IG scheinen bei der Analyse der Videosequenz im Bereich des Monitorings eher in der Lage zu sein, auf ihr vorhandenes pädagogisch-psychologisches Wissens zurückzugreifen und sowohl auf der Stufe des Vorhersagens zu operieren als auch Handlungsalternativen für den weiteren Verlauf zu entwickeln. Im Bereich der prozessualen Strukturierung sowie bei Regeln und Routinen zeigt die prozentuale Verteilung sowie der Median, dass auch in diesen Bereichen die Studierenden der IG im Posttest häufiger beschreiben, interpretieren und Handlungsalternativen generieren als die Studierenden der IG. Diese Unterschiede zwischen den Gruppen sind jedoch nicht signifikant. Die Ergebnisse zeigen zudem, dass nicht alle Studierenden aus der IG ihre wissensgesteuerte Verarbeitung von Unterricht verbessern konnten. Einige Studierende aus der IG verblieben auch im Posttest zumindest bei den Bereichen prozessuale Strukturierung und Regeln und Routinen auf der Ebene des Beschreibens. Da Kompetenz- und Wissensaufbau nicht nur abhängig von den angebotenen Lerngelegenheiten ist, sondern auch von deren individueller Nutzung (Kunina-Habenicht et al. 2013), sollten individuelle Erwerbsprozesse der professionellen Wahrnehmung von Unterricht in zukünftigen Studien stärker in den Blick genommen werden. Als besonders relevant für eine videobasierte Intervention im Lehramtsstudium erachten wir die Fokussierung auf eine Basisdimension guten Unterrichts (in diesem Fall Klassenführung) sowie eine gezielte Strukturierung der videobasierten Reflexionen und des Feedbacks im Sinne der Drei-Schritt-Analyse (siehe auch Kleinknecht und Schneider 2013; Kleinknecht und Gröschner 2016). Dennoch sollte in zukünftigen Studien weiter untersucht werden, ob sich die professionelle Wahrnehmung von Studierenden z. B. durch die Verwendung von Prompts oder noch stärker strukturierte Lernumgebungen deutlicher verbessern lässt.

\subsection{Limitationen}

Zwar können die Ergebnisse der vorliegenden Studie bestätigen, dass sich videogestützte Lerngelegenheiten in Verbindung mit Expertenfeedback positiv auf die professionelle Wahrnehmung von Klassenführung auswirken; allerdings lässt die Auswertung keine endgültigen Aussagen darüber zu, welche Faktoren für die Verbesserungen ausschlaggebend sind. Durch die freiwillige und dadurch nicht rando- 
misierte Teilnahme an der videobasierten Intervention könnten etwa motivationale Aspekte oder Unterschiede im professionellen Wissen die Ergebnisse beeinflusst haben. In der vorliegenden Studie wurde das deklarative Wissen nur in Form der Integration von Einzelereignissen in Fachkonzepte gemessen. Expertinnen und Experten scheint diese Art der Klassifikation aufgrund ihres komplexeren theoretischen Wissens leichter zu fallen (Schwindt 2008), jedoch liefern die vorliegenden Ergebnisse nur bedingt Kenntnisse über den tatsächlichen professionellen Wissensstand der Studierenden. Allerdings zeigten sich in der Stichprobenanalyse nur signifikante Unterschiede im Prästest in Bezug auf Monitoring zugunsten der KG. Dies kehrte sich durch die Intervention in Richtung der IG um. Bei den anderen Variablen zeigten sich keine Prätestunterschiede. Da professionelles Wissen beeinflusst, was wahrgenommen wird und wie die wahrgenommenen Unterrichtsaspekte verarbeitet werden, sollten zukünftige Studien detaillierter untersuchen, wie sich motivationale und wissensspezifische Aspekte auf den Erwerb professioneller Wahrnehmung von Unterricht bei Lehramtsstudierenden auswirken. Eine weitere Limitation betrifft die Messung der selektiven Wahrnehmung. Hier sollten in zukünftigen Studien gezielte „tagging tools" (Walkoe et al. 2019) oder andere Methoden verwendet werden, um mehr Aussagen darüber treffen zu können welche Ereignisse Studierenden wahrnehmen und wie sich diese Wahrnehmung durch eine videobasierte Intervention verändern lässt.

\subsection{Ausblick}

Die Ergebnisse der vorliegenden Studie deuten darauf hin, dass videogestützte Lernformate in Verbindung mit Expertenfeedback einen positiven Einfluss auf die Entwicklung professioneller Wahrnehmung von Lehramtsstudierenden haben. Bezogen auf die Ergebnisse der standardisierten Items zu den Bereichen Beschreibung und Interpretation von Unterrichtssituationen (Weber et al. 2018) konnten noch tiefergehende Erkenntnisse gewonnen werden im Hinblick auf eine Verbesserung der selektiven Wahrnehmung, dem Umgang mit Wertung, dem Generieren von Handlungsalternativen sowie der Klassifikation deklarativer Wissensbestände in beiden Gruppen. Insgesamt liefert die Studie wichtige Erkenntnisse zur Erfassung verschiedener Subprozessen professioneller Wahrnehmung und betont hierdurch auch die Relevanz weniger standardisierter Messverfahren. Hoch standardisierte Verfahren differenzieren bislang zwischen Beschreiben und Interpretieren (Gold und Holodynski 2017) oder Beschreiben, Erklären und Vorhersagen (Seidel et al. 2010). Eine inhaltsanalytische Analyse offener Antwortformate stellt somit eine sinnvolle Ergänzung zu standardisierten Verfahren dar. Insbesondere die Formulierung von Handlungsalternativen ist ein relevanter Subprozess der wissensgesteuerten Verarbeitung von Unterricht (Janik et al. 2013) und bedarf einer inhaltsanalytischen Analyse von offenen Aufgabenformaten (z. B. Sherin und van Es 2009; Kleinknecht und Schneider 2013).

Offen bleibt die Frage, wie die hier gemessene professionelle Wahrnehmung mit dem pädagogisch-psychologischen Wissen, motivationalen Faktoren sowie mit der tatsächlichen Handlungskompetenz der Studierenden zusammenhängt. In dem theoretischen Modell von Blömeke et al. (2015) wird die Transformation von professio- 
nellem Wissen in Performanz über situationsbezogene Fähigkeiten der professionellen Wahrnehmung vermittelt. Hier wären weiterführende Studien von Interesse, die das theoretische Modell von Blömeke et al. (2015) in seiner Wirksamkeit empirisch überprüfen und die Bedeutung professioneller Wahrnehmung von Unterricht für die konkrete Unterrichtsperformanz analysieren.

Funding Open Access funding provided by Projekt DEAL.

Open Access Dieser Artikel wird unter der Creative Commons Namensnennung 4.0 International Lizenz veröffentlicht, welche die Nutzung, Vervielfältigung, Bearbeitung, Verbreitung und Wiedergabe in jeglichem Medium und Format erlaubt, sofern Sie den/die ursprünglichen Autor(en) und die Quelle ordnungsgemäß nennen, einen Link zur Creative Commons Lizenz beifügen und angeben, ob Änderungen vorgenommen wurden.

Die in diesem Artikel enthaltenen Bilder und sonstiges Drittmaterial unterliegen ebenfalls der genannten Creative Commons Lizenz, sofern sich aus der Abbildungslegende nichts anderes ergibt. Sofern das betreffende Material nicht unter der genannten Creative Commons Lizenz steht und die betreffende Handlung nicht nach gesetzlichen Vorschriften erlaubt ist, ist für die oben aufgeführten Weiterverwendungen des Materials die Einwilligung des jeweiligen Rechteinhabers einzuholen.

Weitere Details zur Lizenz entnehmen Sie bitte der Lizenzinformation auf http://creativecommons.org/ licenses/by/4.0/deed.de.

\section{Literatur}

Barth, V.L. (2017). Professionelle Wahrnehmung von Störungen im Unterricht. Wiesbaden: Springer VS.

Berliner, D.C. (2001). Learning about and learning from expert teachers. International Journal of Educational Research, 35, 463-482.

Besa, K.-T., \& Büdcher, M. (2014). Empirische Ergebnisse zur Wirksamkeit von Schulpraktika: Eine Übersicht zum Forschungsstand. In K.-H. Arnold, A. Gröschner \& T. Hascher (Hrsg.), Schulpraktika in der Lehrerbildung/ Pedagogical field experiences in teacher education (S. 129-146). Münster: Waxman.

Blomberg, G., Sherin, M. G., Renkl, A., Glogger, I., \& Seidel, T. (2014). Understanding video as a tool for teacher education: investigating instructional strategies to promote reflection. Instructional Science, 42(3), 433-463.

Blomberg, G., Stürmer, K., \& Seidel, T. (2011). How pre-service teachers observe teaching on video: effects of viewers' teaching subjects and the subject of the video. Teaching and Teacher Education, $27,1131-1140$.

Blömeke, S. (2002). Universität und Lehrerbildung. Bad Heilbrunn: Julius Klinkhardt.

Blömeke, S., Gustafsson, J.-E., \& Shavelson, R. J. (2015). Beyond dichotomies: competence viewed as a continuum. Zeitschrift für Psychologie, 223(1), 3-13.

Blömeke, S., König, J., Busse, A., Suhl, U., Benthien, J., Döhrmann, M., \& Kaiser, G. (2014). Von der Lehrerbildung in den Beruf - Fachbezogenes Wissen als Voraussetzung für Wahrnehmung, Interpretation und Handeln im Unterricht. Zeitschrift für Erziehungswissenschaft, 17(3), 509-542.

Bromme, R. (1992). Der Lehrer als Experte. Zur Psychologie des professionellen Wissens. Bern: Huber.

Cohen, J. (1988). Statistical power analysis for the behavioral sciences (2. Aufl.). Hillsdale: Erlbaum.

Van Es, E. A., \& Sherin, M. G. (2008). Mathematics teachers' „learning to notice“ in the context of a video club. Teaching and Teacher Education, 24, 244-276.

Globalpark, Unipark [Computer Software] (2011). Unipark. http://www.unipark.de. Zugegriffen: Oktober 2017

Gold, B., \& Holodynski, M. (2017). Using digital video to measure the professional vision of elementary classroom management: Test validation and methodological challenges. Computers \& Education, 107, 13-30.

Gold, B., Förster, S., \& Holodynski, M. (2013). Evaluation eines videobasierten Trainingsseminars zur Förderung der professionellen Wahrnehmung von Klassenführung im Grundschulunterricht. Zeitschrift für Pädagogische Psychologie, 27(3), 141-155. 
Gold, B., Hellermann, C., \& Holodynski, M. (2017). Effekte videobasierter Trainings zur Förderung der Selbstwirksamkeitsüberzeugungen über Klassenführung im Grundschulunterricht. Zeitschrift für Erziehungswissenschaft, 20(1), 115-136.

Hellermann, C., Gold, B., \& Holodynski, M. (2015). Förderung von Klassenführungsfähigkeiten im Lehramtsstudium. Zeitschrift für Entwicklungspsychologie und Pädagogische Psychologie, 47(2), 97-109.

Helmke, A., \& Helmke, T. (2014). Wie wirksam ist gute Klassenführung? Lernende Schule, 17(65), 9-12.

Jahn, G., Stürmer, K., Seidel, T., \& Prenzel, M. (2014). Professionelle Unterrichtswahrnehmung von Lehramtsstudierenden - Eine Scaling-up Studie des Observe-Projekts. Zeitschrift für Entwicklungspsychologie und Pädagogische Psychologie, 46(4), 171-180.

Janik, T., Minarikova, E., \& Najvar, P. (2013). Der Einsatz von Videotechnik in der Lehrerbildung. Eine Übersicht leitender Ansätze. In U. Riegel \& K. Macha (Hrsg.), Videobasierte Kompetenzforschung in den Fachdidaktiken. Fachdidaktische Forschungen (S. 63-78). Münster: Waxmann.

Kersting, N. B., Givvin, K. B., Thompson, B. J., Santagata, R., \& Stigler, J. W. (2012). Measuring usable knowledge: teachers' analyses of mathematics classroom videos predict teaching quality and student learning. American Educational Research Journal, 49(3), 568-589.

Kleinknecht, M., \& Gröschner, A. (2016). Fostering preservice teachers' noticing with structured video feedback: results of an online- and video-based intervention study. Teaching and Teacher Education, 59, 45-56.

Kleinknecht, M., \& Schneider, J. (2013). What do teachers think and feel when analyzing videos of themselves and other teachers teaching? Teaching and Teacher Education, 33, 13-23.

Kounin, J. S. (1976). Techniken der Klassenführung. Bern: Huber.

Kramer, C., König, J., Kaiser, G., Ligtvoet, R., \& Blömeke, S. (2017). Der Einsatz von Unterrichtsvideos in der universitären Ausbildung: Zur Wirksamkeit video- und transkriptgestützter Seminare zur Klassenführung auf pädagogisches Wissen und situationsspezifische Fähigkeiten angehender Lehrkräfte. Zeitschrift für Erziehungswissenschaft, 20(1), 137-164.

Kunina-Habenicht, O., Schulze-Stocker, F., Kunter, M., Baumert, J., Leutner, D., Förster, D., Lohse-Bossenz, H., \& Terhart, E. (2013). Die Bedeutung der Lerngelegenheiten im Lehramtsstudium und deren individuelle Nutzung für den Aufbau des bildungswissenschaftlichen Wissens. Zeitschrift für Pädagogik, 59(1), 1-24.

Kunter, M., Klusmann, U., Baumert, J., Richter, D., Voss, T., \& Hachfeld, A. (2013). Professional competence of teachers: effects on instructional quality and student development. Journal of Educational Psychology, 105, 805-820.

Lamnek, S. (1995). Qualitative Sozialforschung. Weinheim: Beltz.

Landis, J., \& Koch, G. (1977). The measurement of observer agreement for categorical data. Biometrics, $33,159-174$.

Lindmeier, A. (2013). Video-vignettenbasierte standardisierte Erhebung von Lehrerkognitionen. In U. Riegel \& K. Macha (Hrsg.), Videobasierte Kompetenzforschung in den Fachdidaktiken. Fachdidaktische Forschungen (S. 45-62). Münster: Waxmann.

Rich, P., \& Hannafin, M. J. (2008). Capturing and assessing evidence of student teacher inquiry: A case study. Teaching and Teacher Education, 24(6), 1426-1440.

Santagata, R., \& Guarino, J. (2011). Using video to teach future teachers to learn from teaching. ZDM, 43, $133-145$.

Schönbächler, M.T. (2008). Klassenmanagement. Situative Gegebenheiten und personale Faktoren in Lehrpersonen- und Schülerperspektive. Bern: Haupt.

Schwindt, K. (2008). Lehrpersonen betrachten Unterricht. Kriterien für die kompetente Unterrichtswahrnehmung. Münster: Waxmann.

Seidel, T. (2009). Klassenführung. In E. Wild \& J. Möller (Hrsg.), Pädagogische Psychologie (S. 136-148). Heidelberg: Springer.

Seidel, T., \& Prenzel, M. (2007). Wie Lehrpersonen Unterricht wahrnehmen und einschätzen - Erfassung pädagogisch-psychologischer Kompetenzen bei Lehrpersonen mit Hilfe von Videosequenzen. In M. Prenzel, I. Gogolin \& H.-H. Krüger (Hrsg.), Kompetenzdiagnostik (Zeitschrift für Erziehungswissenschaft: Sonderheft 8, S. 201-218). Wiesbaden: Springer VS.

Seidel, T., \& Stürmer, K. (2014). Modeling and measuring the structure of professional vision in preservice teachers. American Educational Research Journal, 51(4), 739-771.

Seidel, T., \& Thiel, F. (2017). Standards und Trends der videobasierten Lehr- Lernforschung. Zeitschrift für Erziehungswissenschaft, 20(1), 1-21.

Seidel, T., Blomberg, G., \& Stürmer, K. (2010). „Observer“: Validierung eines videobasierten Instruments zur Erfassung der professionellen Wahrnehmung von Unterricht. Projekt OBSERVE. Zeitschrift für Pädagogik, 56, 296-306. 
Seidel, T., Stürmer, K., Blomberg, G., Kobarg, M., \& Schwindt, K. (2011). Teacher learning from analysis of videotaped classroom situations: Does it make a difference whether teachers observe their own teaching or that of others? Teaching and Teacher Education, 27(2), 259-267.

Sherin, M. G. (2007). The development of teachers' professional vision in video clubs. In R. Goldman, R. Pea, B. Barron \& S. Derry (Hrsg.), Video research in the learning sciences (S. 383-395). Hillsdale: Erlbaum.

Sherin, M. G., \& van Es, E. A. (2009). Effects of video club participation on teachers' professional vision. Journal of Teacher Education, 60(1), 20-37.

Stürmer, K. (2011). Voraussetzungen für die Entwicklung Professioneller Unterrichtswahrnehmung im Rahmen universitärer Lehrerbildung. Dissertation. München: Technische Universität München.

Stürmer, K., Seidel, T., \& Kunina-Habenicht, O. (2015). Unterricht wissensbasiert beobachten: Unterschiede und erklärende Faktoren bei Referendaren zum Berufseinstieg. Zeitschrift für Pädagogik, 61(3), 345-359.

Thiel, F., Richter, S., \& Ophardt, D. (2012). Steuerung von Übergängen im Unterricht - Eine ExpertenNovizen-Studie zum Klassenmanagement. Zeitschrift für Erziehungswissenschaft, 15(4), 727-752.

Tripp, T. R., \& Rich, P. J. (2012). The influence of video analysis on the process of teacher change. Teaching and Teacher Education, 28(5), 728-739.

Walkoe, J., Sherin, M. G., \& Elby, A. (2019). Video tagging as a window into teacher noticing. Journal of Mathematics Teacher Education. https://doi.org/10.1007/s10857-019-09429-0.

Weber, K.E., Gold, B., Prilop, C. N., \& Kleinknecht, M. (2018). Promoting pre-service teachers' professional vision of classroom management during practical school training: effects of a structured online- and video-based self-reflection and feedback intervention. Teaching and Teacher Education, 76, 39-49.

Wirtz, M., \& Caspar, F. (2002). Beurteilerübereinstimmung und Beurteilerreliabilität: Methoden zur Bestimmung und Verbesserung der Zuverlässigkeit von Einschätzungen mittels Kategoriensystemen und Ratingskalen. Göttingen: Hogrefe.

Wolff, C.E., Jarodzka, H., \& Boshuizen, H. (2017). See and tell: Differences between expert and novice teachers' interpretations of problematic classroom management events. Teaching and Teacher Education, 66, 295-308. 